\title{
Uso da anticoncepção de emergência entre mulheres usuárias de Unidades Básicas de Saúde em três capitais brasileiras
}

\author{
Emergency contraceptive use among women attending Primary \\ Health Care services in three Brazilian capital cities
}

\author{
Ana Luiza Vilela Borges (https://orcid.org/0000-0002-2807-1762) ${ }^{1}$ \\ Renata Ferreira Sena Gonçalves (https://orcid.org/0000-0001-8370-3948) ${ }^{1}$ \\ Christiane Borges do Nascimento Chofakian (https://orcid.org/0000-0002-5953-3296) ${ }^{2}$ \\ Natália de Castro Nascimento (https://orcid.org/0000-0003-2498-5281) ${ }^{1}$ \\ Regina Maria Mac Dowell de Figueiredo (https://orcid.org/0000-0001-9880-7045) ${ }^{3}$ \\ Elizabeth Fujimori (https://orcid.org/0000-0002-7991-0503) ${ }^{1}$ \\ Osmara Alves dos Santos (https://orcid.org/0000-0001-6360-861X) ${ }^{1}$ \\ Eveline Do Amor Divino (https://orcid.org/0000-0003-0037-0557) ${ }^{4}$
}

${ }^{1}$ Departamento de Enfermagem em Saúde Coletiva, Escola de

Enfermagem, Universidade de São Paulo (USP). R. Dr.

Enéias de Carvalho Aguiar 419, Cerqueira César. 05403-000 São Paulo SP Brasil. alvilela@usp.br

${ }^{2}$ Escola de Artes, Ciências e Humanidades, USP. São

Paulo SP Brasil.

${ }^{3}$ Instituto de Saúde,

Secretaria de Estado da

Saúde de São Paulo. São

Paulo SP Brasil.

${ }^{4}$ Faculdade de Enfermagem,

Universidade Federal de

Mato Grosso. Cuiabá MT

Brasil.

\begin{abstract}
Little is known regarding the use of emergency contraception among women from different regions of Brazil. The use of emergency contraception as well as contraceptive methods before and after coitus was analyzed. This cross-sectional study assessed the use of emergency contraception by interviewing 2,051 women aged between 18 and 49 attending 76 basic health units in three capitals: São Paulo-SP, Aracaju-SE and Cuiabá-MT. Aspects associated with the use of emergency contraception were analyzed by means of multiple logistic regression. Over half of the women reported the use of emergency contraception (56.7\%). Having a high level of education, being from a more privileged socioeconomic group, having a paid job and having had four or more sexual partners were associated with the use of emergency contraception. Being 35 years of age or older and being in a stable relationship was negatively associated. The last time they used emergency contraception, $53.2 \%$ used another method, with the male condom and oral pill being the most frequent. Of those who did not use the method, half adopted the regular method after using it (51.7\%). The conclusion drawn is that emergency contraception is widely used and does not appear to affect the use of the regular contraceptive method.

Key words Postcoital contraception, Sexual and reproductive health, Women's health, Family planning
\end{abstract}

Resumo Pouco se sabe sobre o uso da anticoncepção de emergência entre mulheres de diferentes regiões do país. Este estudo analisou o uso da anticoncepção de emergência e os aspectos associados, bem como o uso de métodos contraceptivos antes e após. Trata-se de estudo transversal, conduzido com 2.051 mulheres de 18-49 anos, usuárias de 76 Unidades Básicas de Saúde de São Paulo-SP, Aracaju-SE e Cuiabá-MT. Os aspectos associados ao uso da anticoncepção de emergência foram analisados por meio de regressão logística múltipla. Mais da metade das mulheres relatou já ter usado a anticoncepção de emergência (56,7\%). Ter alta escolaridade, ser de grupo socioeconômico mais favorecido, ter trabalho remunerado e ter tido quatro ou mais parceiros sexuais associou-se com uso de anticoncepção de emergência. Ter 35 anos de idade ou mais e estar em união estável associou-se negativamente. Da última vez que usaram a anticoncepção de emergência, $53,2 \%$ usavam outro método, sendo preservativo masculino e pílula oral os mais frequentes. Das que não usavam método, metade adotou método regular após o uso (51,7\%). Conclui-se que a anticoncepção de emergência é amplamente utilizada e parece não contribuir para interrupção do método contraceptivo de uso regular.

Palavras-chave Anticoncepção Pós-Coito, Saúde Sexual e Reprodutiva, Saúde da Mulher, Planejamento familiar 


\section{Introdução}

A anticoncepção de emergência (AE) constituise em um conjunto de métodos anticonceptivos indicados para serem usados para prevenir a gestação após uma relação sexual desprotegida. Atualmente, existem cinco tipos de produtos que atuam como AE: o método Yuzpe, a pílula de levonorgestrel, o DIU de cobre, a pílula de acetato de ulipristal e a pílula de mifepristona ${ }^{1,2}$. Pelo fato de a AE se constituir como a única estratégia existente para prevenir uma gravidez após a relação sexual, o acesso a este insumo constitui-se parte indispensável dos direitos das mulheres, ancorado nos direitos sexuais e reprodutivos. Certamente, a possibilidade de utilizá-la, quando indicada, é um recurso a mais para mulheres e casais prevenirem uma gravidez não intencional em situações de falha ou de não uso de método contraceptivo de rotina ou em casos de violência sexual ${ }^{3}$.

A pílula de levonorgestrel está disponível em mais de 140 países $^{4}$ e é a única opção de AE disponível no Brasil (embora o dispositivo intrauterino com cobre seja também reconhecido como um método de contracepção de emergência, não é recomendado nessa situação no Brasil). É disponibilizada sem receita médica na maioria dos países, o que permite o acesso rápido e fácil à AE nas farmácias comerciais 5 . Seu uso deve ser feito até 120 horas (5 dias) após a relação sexual desprotegida; porém, sua eficácia é maior quanto antes for utilizada ${ }^{6}$. No Brasil, a AE na apresentação de 1,5 mg de levonorgestrel é aprovada pelo Ministério da Saúde e está disponível tanto em farmácias comerciais quanto nos serviços públicos de saúde, como Unidades Básicas de Saúde (UBS), incluindo as que adotam a Estratégia Saúde da Família (ESF), e em Unidade de Pronto Atendimento (UPA), onde é disponibilizada sem que haja necessidade de receita médica ${ }^{6}$, podendo ser prescrita por enfermeiros ${ }^{3}$.

De acordo com última Pesquisa Nacional de Demografia e Saúde de 20067, a AE foi utilizada alguma vez na vida por $12 \%$ das mulheres entre 15 e 49 anos pesquisadas, sendo seu uso mais frequente entre as jovens de 20 a 24 anos de idade (18,5\%). Nos últimos anos, estudos de abrangência local têm mostrado uma porcentagem maior de uso da AE entre estudantes do Ensino Médio do município de São Paulo $(30,1 \%)^{8}$, entre estudantes do Ensino Médio de cidade da Região Metropolitana de São Paulo $(57,9 \%)^{9}$ e entre adolescentes do município de São Paulo $(60,0 \%)^{10}$. Da mesma forma, estudo realizado com jovens uni- versitárias de Santa Catarina mostrou que 48,6\% já haviam usado a $\mathrm{AE}^{11}$, resultado similar ao observado entre jovens universitárias do estado de São Paulo $(50,4 \%)^{12}$. Outro estudo, conduzido com gestantes de 12 UBS do município de São Paulo revelou que 55,7\% das mulheres já haviam utilizado a $\mathrm{AE}^{13}$.

Considerando que adolescentes ainda constituem o grupo mais estudado no Brasil em relação à $\mathrm{AE}^{14}$ e que pouco se sabe sobre sua utilização e fatores associados entre mulheres de diferentes regiões do Brasil, bem como sobre o uso de métodos contraceptivos antes e após o uso da $\mathrm{AE}$, este estudo foi conduzido com o objetivo de analisar o uso da $\mathrm{AE}$ em três capitais brasileiras e os aspectos associados, bem como descrever a dinâmica do uso de métodos contraceptivos antes e após o uso da AE.

\section{Método}

Este estudo quantitativo do tipo transversal integrou uma investigação mais ampla intitulada "Padrões e determinantes das descontinuidades contraceptivas e o papel da anticoncepção de emergência", o qual avaliou as práticas sexuais, contraceptivas e reprodutivas nos últimos cinco anos de vida da mulher. Foi conduzido em amostra probabilística de mulheres de 18 a 49 anos de idade, usuárias de UBS de três capitais de estado de diferentes regiões do país: São Paulo-SP, Aracaju-SE e Cuiabá-MT

Para determinar o tamanho da amostra em cada município, utilizou-se como parâmetro, a proporção de mulheres a ser estimada igual a $50 \%$; nível de confiança de 95\%; erro de amostragem igual a $5 \%$; e efeito do delineamento (deff) igual a 2 . O cálculo indicou tamanho amostral de 768 mulheres em São Paulo, 385 em Aracaju e 385 em Cuiabá. Como as estimativas deveriam ser obtidas entre mulheres que usaram algum método contraceptivo, proporção que no Brasil é estimada em $80 \%^{7}$, o número de mulheres a serem entrevistadas passou para 1.000 em São Paulo, 482 em Aracaju e 482 em Cuiabá. Considerou-se também o percentual de $25 \%$ de mulheres que não responderiam ao questionário (recusa ou perda por problemas do entrevistador) e de 33\% de mulheres que não seriam elegíveis para a entrevista (critérios de exclusão foram nunca ter tido relação sexual e mulheres laqueadas/parceiros vasectomizados há mais de cinco anos $)^{7}$. Dessa forma, deveriam ser selecionadas 1.993 mulheres para a cidade de São Paulo, com o 
intuito de se obter 1.000 entrevistas válidas, bem como 963 mulheres em Aracaju e 963 em Cuiabá, para se obter pelo menos 482 entrevistas válidas em cada uma dessas capitais.

O plano amostral foi conduzido por meio de amostragem por conglomerados, em duas etapas. Na primeira etapa, as UBS foram sorteadas com probabilidade proporcional ao tamanho, medido pelo número de exames citopatológicos cérvicovaginais realizados em 2014. Por esse critério, 78 UBS foram selecionadas: 38 em São Paulo, do total de 441 UBS; 19 em Aracaju, dentre 43; e 19 em Cuiabá, dentre 93. Na segunda etapa, foram selecionadas por amostragem não probabilística as mulheres a serem entrevistadas em cada UBS, de acordo com os seguintes critérios: 1) mulheres que aguardavam atendimento para realização do exame citopatológico cérvico-vaginal; 2) mulheres que aguardavam consulta médica ou de enfermagem; 3) mulheres que aguardavam atendimento para qualquer outra atividade na UBS. Nas três capitais, a pesquisa foi realizada em três dias consecutivos em cada UBS e, em cada dia, foram entrevistadas nove mulheres, totalizando 27 entrevistas válidas por dia por UBS. Por fim, foram obtidas 1.030 entrevistas válidas em São Paulo, 508 em Aracaju e 513 em Cuiabá, totalizando 2.051 mulheres.

A coleta de dados foi realizada por meio de entrevista face a face por pesquisadoras graduadas na área da saúde (enfermeiras, psicólogas e obstetrizes) e durou cerca de 20 minutos. As mulheres selecionadas foram abordadas pelas entrevistadoras e convidadas a participar da pesquisa nas UBS. Foram explicados os objetivos, o conteúdo das questões e as etapas da entrevista. As mulheres que aceitaram participar da pesquisa assinaram o Termo de Consentimento Livre e Esclarecido. As entrevistas foram realizadas com o uso de instrumento estruturado na plataforma Census and Survey Processing System (CSPro) em tablets. Em São Paulo-SP, a coleta de dados ocorreu de outubro a dezembro de 2015; em AracajuSE, entre agosto e setembro de 2016; e em Cuiabá -MT, de agosto a setembro de 2017.

Foram obtidas informações sobre o perfil sociodemográfico, o comportamento sexual e história reprodutiva das mulheres. O perfil sociodemográfico foi composto pelas variáveis idade (18-24 anos, 25-34 anos e 35 anos e mais), escolaridade (até 8 anos, 9-11 anos e 12 anos e mais), cor de pele autoclassificada (branca, parda, preta, amarela/indígena), grupo socioeconômico (A e B, C, D e E, segundo o Critério de Classificação Econômica Brasil, da Associação Brasileira de
Empresas de Pesquisa, sendo A referente ao grupo com maior possibilidade de consumo e E o grupo com a menor possibilidade de consumo), religião (católica, evangélica, nenhuma e outras, que incluíram as religiões espírita, judia, umbandista, candomblé), trabalho remunerado (não e sim) e plano de saúde (não e sim). O comportamento sexual e a história reprodutiva foram compostos pelas variáveis idade na primeira relação sexual (<14 anos, 15-18 anos e 19 anos e mais), número de parceiros sexuais (um, dois a três e quatro e mais), união conjugal (não e sim, esta última incluindo união formal e consensual), número de filhos (nenhum, um, dois, três e mais) e aborto anterior (não e sim).

Para analisar a dinâmica contraceptiva antes e após o uso da AE, foram consideradas apenas as 832 mulheres que relataram ter usado a AE nos cinco anos anteriores à entrevista. Isso foi necessário para minimizar o viés de memória. Essas mulheres foram questionadas sobre qual método contraceptivo utilizavam na última vez em que usaram a AE (camisinha masculina, pílula oral, injetável, adesivo, dispositivo intrauterino e métodos naturais, como tabelinha, coito interrompido e sintotérmico) e qual foi o método contraceptivo adotado após o uso da AE (idem, com a inclusão da esterilização).

A análise estatística foi realizada utilizando o Stata 14.2. Os fatores associados ao uso anterior da $\mathrm{AE}$ foram analisados por meio do teste de diferença de proporções pelo Qui-quadrado ou Exato de Fisher. Essas análises foram conduzidas, primeiramente, de forma separada por capitais e, posteriormente, com os dados agregados. Depois, foi realizada a regressão logística múltipla com todas as variáveis sociodemográficas e da história reprodutiva, inseridas simultaneamente no modelo, tendo como variável dependente o uso anterior da AE (não/sim). Os dados sobre a dinâmica contraceptiva foram analisados por meio de número absoluto e proporções.

O projeto foi aprovado por Comitê de Ética em Pesquisa credenciado.

\section{Resultados}

Dentre as 2.051 mulheres entrevistadas, a idade média foi 30,1 anos $(\mathrm{dp}=7,9)$, sendo que um terço da amostra foi composta por mulheres jovens (18 a 24 anos). Aproximadamente metade autodeclarou-se parda $(52,3 \%)$, apenas $14,7 \%$ das mulheres estudaram 12 anos ou mais, a maioria pertencia ao grupo socioeconômico C $(63,8 \%)$, 
tinha trabalho remunerado $(52,0 \%)$ e não possuía plano de saúde $(82,8 \%)$. A maior parte das mulheres teve a primeira relação sexual entre os 15 e os 18 anos (62,7\%), estava em união conjugal no momento da entrevista $(73,6 \%)$, tinha filhos $(87,0 \%)$ e não relatou aborto anterior $(73,3 \%)$ (Tabela 1$)$.

No geral, mais da metade das mulheres entrevistadas $(56,7 \%)$ relatou já ter usado a $\mathrm{AE}$ anteriormente: 56,4\% em São Paulo, 64,3\% em Cuiabá e 49,8\% em Aracaju (dados não apresentados em tabela). Os aspectos associados ao uso anterior da $\mathrm{AE}$ foram o município de residência $(p<0,001)$, a idade $(p<0,001)$, a escolaridade $(\mathrm{p}<0,001)$, o grupo socioeconômico $(\mathrm{p}<0,001)$, a religião $(\mathrm{p}<0,001)$, o trabalho remunerado $(\mathrm{p}=0,029)$, a idade na primeira relação sexual $(\mathrm{p}<0,001)$, o número de parceiros sexuais $(\mathrm{p}<0,001)$, a união conjugal $(\mathrm{p}<0,001)$ e o número de filhos $(\mathrm{p}<0,001)$ (Tabela 1).

A Tabela 2 apresenta os modelos de regressão logística simples e múltipla, segundo as variáveis associadas ao uso anterior da AE. Os resultados da análise de regressão logística múltipla mostraram que, comparadas às mulheres de 18 a 24 anos de idade, mulheres de 25-34 $(\mathrm{OR}=0,59 ; \mathrm{IC} 95 \%=0,44-0,79)$ e com mais de 35 anos $(\mathrm{OR}=0,21$; IC95\% $=0,14-0,30)$ tiveram menos chance de usar a AE. As mulheres unidas também mostram menos chance de usar AE em comparação às mulheres não unidas $(\mathrm{OR}=0,61$; IC95\% $=0,46-0,80)$. Por sua vez, mulheres mais escolarizadas $\quad(\mathrm{OR}=2,63 ; \quad \mathrm{IC} 95 \%=1,76-3,94)$, pertencentes a grupos socioeconômicos mais favorecidos $(\mathrm{OR}=1,92$; IC95\%=1,27-2,90), com trabalho remunerado $(\mathrm{OR}=1,25$; IC $95 \%=1,01$ $1,57)$ e quatro ou mais parceiros sexuais ao longo da vida $(\mathrm{OR}=4,53$; $\mathrm{IC} 95 \%=3,33-6,17)$ foram as que tiveram mais chance de usar a AE. Não houve diferença estatisticamente significativa entre usar a AE e ser usuária das UBS nas três capitais consideradas.

Os resultados quanto ao método contraceptivo utilizado pelas mulheres que haviam usado $\mathrm{AE}$ nos cinco anos anteriores à entrevista $(\mathrm{n}=832)$ encontram-se na Figura 1. Constata-se que pouco mais da metade das mulheres $(53,2 \%)$ utilizava método contraceptivo quando usou a $\mathrm{AE}$, sendo a camisinha masculina $(39,7 \%)$ e a pílula oral $(38,6 \%)$ os mais citados.

A dinâmica contraceptiva após o uso da $\mathrm{AE}$ está representada na Figura 2. Observa-se que, dentre as mulheres que utilizavam a camisinha masculina quando usaram da AE, 80,6\% referiram ter mantido sua utilização. Por sua vez,
90,6\% das mulheres que utilizavam a pílula oral continuaram a utilizá-la; e $88,7 \%$ das mulheres usuárias de injetável mantiveram esse método contraceptivo após o uso da AE. Ou seja, o uso da $\mathrm{AE}$ não contribuiu para que a maioria das mulheres interrompesse ou trocasse o método contraceptivo. No que concerne às mulheres que relataram estar sob uso de métodos naturais quando usaram a $\mathrm{AE}$, mais da metade continuou a usar o mesmo método (58,5\%), porém $19,5 \%$ adotaram um método mais eficaz posteriormente, sendo eles o injetável, a pílula oral e a camisinha masculina (dado não mostrado na Figura). Duas mulheres que relataram usar adesivo continuaram usando-o após o uso da $\mathrm{AE}$ (dado não mostrado na Figura).

Dentre as 387 mulheres que não usavam método contraceptivo quando utilizaram a $\mathrm{AE}$, metade passou a adotar método contraceptivo regular $(51,7 \%)$. Dentre essas, a maior parte iniciou o uso da pílula oral $(43,0 \%)$ e da camisinha masculina (30,5\%), seguida do injetável $(23,0 \%)$ (Figura 3).

\section{Discussão}

Este estudo descreve o uso da AE entre mulheres de 18 a 49 anos de idade, usuárias de UBS em três capitais de estado de regiões diferentes do Brasil: Sudeste, representada por São Paulo; Nordeste, por Aracaju e região Centro-Oeste, representada por Cuiabá. Os resultados permitiram verificar o uso da $\mathrm{AE}$ entre mulheres e jovens com perfis sociais e culturais heterogêneos que utilizam serviços públicos de saúde da atenção primária, organizados de forma diversa, uma vez que pertencem a diferentes municipalidades.

Constatou-se que praticamente metade das mulheres de diferentes regiões brasileiras relatou ter usado a AE alguma vez na vida. Este achado é similar ao observado por Santos et al. ${ }^{13} \mathrm{em} \mathrm{mu-}$ lheres usuárias de UBS da cidade de São Paulo e, de certa forma, similar aos achados de estudos conduzidos com universitárias ${ }^{11,12}$. Contudo, comparado ao cenário internacional, verifica-se que a proporção de uso da $\mathrm{AE}$ varia de acordo com o período e cenário, mas, em geral, mostrase menor: no México, estudo com mulheres de 15 a 29 anos observou prevalência de $29 \%$ de uso da AE em 2014 ${ }^{15}$; nos EUA, $11 \%$ das mulheres com idade entre 15 e 44 anos já tinham usado a $\mathrm{AE}$ entre 2006 e $2010^{16}$; e na França, 16,9\% das mulheres de 15 a 44 anos referiram já ter usado a $\mathrm{AE}$ em $2004^{17}$. 
Tabela 1. Características sociodemográficas, do comportamento sexual e da história reprodutiva das mulheres usuárias de Unidades Básicas de Saúde de acordo com o uso anterior da anticoncepção de emergência. São PauloSP, Aracaju-SE e Cuiabá-MT, 2015-2017.

\begin{tabular}{|c|c|c|c|c|c|c|c|}
\hline \multirow{3}{*}{ Variável } & \multirow{2}{*}{\multicolumn{2}{|c|}{ Total }} & \multicolumn{5}{|c|}{ Uso anterior da anticoncepção de emergência } \\
\hline & & & \multicolumn{2}{|c|}{ Não } & \multicolumn{2}{|c|}{ Sim } & \multirow[t]{2}{*}{ p } \\
\hline & n & $\%$ & n & $\%$ & n & $\%$ & \\
\hline \multicolumn{8}{|l|}{ Município } \\
\hline São Paulo & 1.030 & 50,2 & 449 & 50,6 & 581 & 49,9 & $<0,001$ \\
\hline Aracaju & 508 & 24,8 & 255 & 28,8 & 253 & 21,7 & \\
\hline Cuiabá & 513 & 25,0 & 183 & 20,6 & 330 & 28,4 & \\
\hline \multicolumn{8}{|l|}{ Idade (anos) } \\
\hline $18-24$ & 607 & 29,6 & 190 & 21,4 & 417 & 35,8 & $<0,001$ \\
\hline $25-34$ & 839 & 40,9 & 320 & 36,1 & 519 & 44,6 & \\
\hline 35 e mais & 605 & 29,5 & 377 & 42,5 & 228 & 19,6 & \\
\hline \multicolumn{8}{|l|}{ Escolaridade (anos) } \\
\hline Até 8 & 555 & 27,1 & 329 & 37,1 & 226 & 19,4 & $<0,001$ \\
\hline $9-11$ & 1.194 & 58,2 & 461 & 52,0 & 733 & 63,0 & \\
\hline 12 e mais & 302 & 14,7 & 97 & 10,9 & 205 & 17,6 & \\
\hline \multicolumn{8}{|l|}{ Cor da Pele* } \\
\hline Branca & 506 & 24,7 & 222 & 25,0 & 284 & 24,4 & 0,222 \\
\hline Parda & 1.072 & 52,3 & 479 & 54,1 & 593 & 51,1 & \\
\hline Preta & 360 & 17,6 & 145 & 16,4 & 215 & 18,5 & \\
\hline Amarela/indígena & 110 & 5,4 & 40 & 4,5 & 70 & 6,0 & \\
\hline \multicolumn{8}{|l|}{ Grupo socioeconômico } \\
\hline A e B & 295 & 14,4 & 107 & 12,1 & 188 & 16,1 & $<0,001$ \\
\hline $\mathrm{C}$ & 1.039 & 63,8 & 549 & 61,9 & 760 & 65,3 & \\
\hline $\mathrm{D}$ e $\mathrm{E}$ & 447 & 21,8 & 231 & 26,0 & 216 & 18,6 & \\
\hline \multicolumn{8}{|l|}{ Religião } \\
\hline Católica & 951 & 46,4 & 453 & 51,1 & 498 & 42,8 & $<0,001$ \\
\hline Evangélica & 751 & 36,6 & 303 & 34,1 & 448 & 35,5 & \\
\hline Nenhuma & 256 & 12,5 & 91 & 10,3 & 165 & 14,2 & \\
\hline Outras ${ }^{\star \star}$ & 93 & 4,5 & 40 & 4,5 & 53 & 4,5 & \\
\hline \multicolumn{8}{|l|}{ Exerce trabalho remunerado } \\
\hline Não & 984 & 48,0 & 450 & 50,7 & 534 & 45,9 & 0,029 \\
\hline Sim & 1.067 & 52,0 & 437 & 49,3 & 630 & 54,1 & \\
\hline \multicolumn{8}{|l|}{ Possui plano de saúde } \\
\hline Não & 1.698 & 82,8 & 749 & 84,4 & 949 & 81,5 & 0,083 \\
\hline Sim & 353 & 17,2 & 138 & 15,6 & 215 & 18,5 & \\
\hline \multicolumn{8}{|c|}{ Idade na primeira relação sexual } \\
\hline$<14$ anos & 347 & 16,9 & 144 & 16,2 & 203 & 17,4 & $<0,001$ \\
\hline $15-18$ & 1.285 & 62,7 & 510 & 57,5 & 775 & 66,6 & \\
\hline 19 e mais & 419 & 20,4 & 233 & 26,3 & 186 & 16,0 & \\
\hline \multicolumn{8}{|c|}{ Número de parceiros sexuais ${ }^{\star}$} \\
\hline Um & 457 & 22,4 & 292 & 33,0 & 165 & 14,2 & $<0,001$ \\
\hline Dois a três & 852 & 41,6 & 402 & 45,5 & 450 & 38,7 & \\
\hline Quatro e mais & 737 & 36,0 & 190 & 21,5 & 547 & 47,1 & \\
\hline \multicolumn{8}{|l|}{ União conjugal } \\
\hline Não & 542 & 26,4 & 176 & 19,8 & 366 & 31,4 & $<0,001$ \\
\hline Sim & 1.509 & 73,6 & 711 & 80,2 & 798 & 68,6 & \\
\hline \multicolumn{8}{|l|}{ Número de filhos } \\
\hline Nenhum & 235 & 13,0 & 73 & 9,2 & 162 & 16,0 & $<0,001$ \\
\hline Um & 747 & 41,4 & 300 & 38,0 & 447 & 44,0 & \\
\hline Dois & 485 & 26,9 & 242 & 30,7 & 243 & 24,0 & \\
\hline Três e mais & 336 & 18,7 & 174 & 22,1 & 162 & 16,0 & \\
\hline Aborto anterior $(\mathrm{n}=1803)$ & & & & & & & \\
\hline Não & 1.333 & 73,3 & 576 & 73,0 & 757 & 74,7 & 0,428 \\
\hline Sim & 470 & 26,1 & 213 & 27,0 & 257 & 25,3 & \\
\hline Total & 2.051 & 100 & 887 & 100,0 & 1.164 & 100,0 & \\
\hline
\end{tabular}

${ }^{\star}$ Duas mulheres se recusaram a responder. ${ }^{* *}$ Outras: espírita, judia, umbandista, candomblé. 
Tabela 2. Análise de regressão logística simples e múltipla, segundo os aspectos associados ao uso anterior da anticoncepção de emergência. São Paulo-SP, Aracaju-SE e Cuiabá-MT, 2015-2017.

\begin{tabular}{|c|c|c|c|c|c|}
\hline \multirow[t]{2}{*}{ Variáveis } & \multirow[t]{2}{*}{ Categorias } & \multicolumn{2}{|c|}{$\begin{array}{c}\text { Regressão } \\
\text { logística simples }\end{array}$} & \multicolumn{2}{|c|}{$\begin{array}{l}\text { Regressão logística } \\
\text { múltipla }\end{array}$} \\
\hline & & ORbruta & IC95\% & ORajust & IC95\% \\
\hline \multirow[t]{3}{*}{ Município } & São Paulo & 1,00 & - & 1,00 & - \\
\hline & Aracaju & 0,76 & $0,61-0,94$ & 0,97 & $0,74-1,27$ \\
\hline & Cuiabá & 1,39 & $1,11-1,73$ & 1,08 & $0,82-1,41$ \\
\hline \multirow{3}{*}{ Idade $^{*}$} & $18-24$ & 1,00 & - & 1,00 & - \\
\hline & $25-34$ & 0,73 & $0,59-0,92$ & 0,59 & $0,44-0,79$ \\
\hline & 35 e mais & 0,27 & $0,21-0,34$ & 0,21 & $0,14-0,30$ \\
\hline \multirow[t]{3}{*}{ Escolaridade $^{*}$} & Até 8 & 1,00 & - & 1,00 & - \\
\hline & $9-11$ & 2,31 & $1,88-2,84$ & 1,74 & $1,35-2,24$ \\
\hline & 12 e mais & 3,07 & $2,29-4,13$ & 2,63 & $1,76-3,94$ \\
\hline \multirow[t]{4}{*}{ Cor da Pele } & Branca & 1,00 & - & 1,00 & - \\
\hline & Parda & 0,96 & $0,78-1,19$ & 0,93 & $0,71-1,22$ \\
\hline & Preta & 1,15 & $0,88-1,52$ & 1,08 & $0,77-1,52$ \\
\hline & Amarela/indígena & 1,36 & $0,89-2,09$ & 1,19 & $0,70-2,02$ \\
\hline \multirow[t]{3}{*}{ Grupo socioeconômico* } & $\mathrm{D}$ e E & 1,00 & - & 1,00 & - \\
\hline & $\mathrm{C}$ & 1,48 & $1,19-1,83$ & 1,40 & $1,07-1,84$ \\
\hline & $\mathrm{A}$ e $\mathrm{B}$ & 1,87 & $1,39-2,53$ & 1,92 & $1,27-2,90$ \\
\hline \multirow[t]{4}{*}{ Religião } & Católica & 1,00 & - & 1,00 & - \\
\hline & Evangélica & 0,60 & $0,45-0,80$ & 0,82 & $0,58-1,16$ \\
\hline & Nenhuma & 0,81 & $0,60-1,09$ & 1,04 & $0,74-1,48$ \\
\hline & Outras $* *$ & 0,73 & $0,45-1,18$ & 0,65 & $0,36-1,19$ \\
\hline \multirow[t]{2}{*}{ Exerce trabalho remunerado* } & Não & 1,00 & - & 1,00 & - \\
\hline & Sim & 1,21 & $1,01-1,44$ & 1,25 & $1,01-1,57$ \\
\hline \multirow[t]{2}{*}{ Possui plano de saúde } & Não & 1,00 & - & 1,00 & - \\
\hline & Sim & 1,22 & $0,97-1,55$ & 0,81 & $0,60-1,09$ \\
\hline \multirow[t]{3}{*}{ Idade na primeira relação sexual } & $<14$ anos & 1,00 & - & 1,00 & - \\
\hline & $15-18^{*}$ & 1,07 & $0,84-1,37$ & 1,33 & $0,99-1,78$ \\
\hline & 19 e mais & 0,56 & $0,42-0,75$ & 1,16 & $0,78-1,72$ \\
\hline \multirow[t]{3}{*}{ Número de parceiros sexuais ${ }^{\star}$} & Um & 1,00 & - & 1,00 & - \\
\hline & Dois a três & 1,98 & $1,56-2,50$ & 1,95 & $1,48-2,57$ \\
\hline & Quatro e mais & 5,09 & $3,95-6,55$ & 4,53 & $3,33-6,17$ \\
\hline \multirow[t]{2}{*}{ União conjugal $^{*}$} & Não & 1,00 & - & 1,00 & - \\
\hline & Sim & 0,53 & $0,43-0,66$ & 0,61 & $0,46-0,80$ \\
\hline \multirow[t]{4}{*}{ Número de filhos } & Nenhum & 1,00 & - & 1,00 & - \\
\hline & Um & 0,67 & $0,49-0,91$ & 1,15 & $0,81-1,65$ \\
\hline & Dois & 0,45 & $0,32-0,62$ & 1,08 & $0,73-1,61$ \\
\hline & Três e mais & 0,41 & $0,29-0,59$ & 1,17 & $0,75-1,81$ \\
\hline \multirow[t]{2}{*}{ Aborto anterior } & Não & 1,00 & - & 1,00 & - \\
\hline & Sim & 0,89 & $0,72-1,10$ & 1,00 & $0,79-1,28$ \\
\hline
\end{tabular}

${ }^{*} \mathrm{p}<0,05 .{ }^{* *}$ Outras religiões incluem: espírita, judia, umbandista, candomblé.

Fonte: Elaborado pelas autoras.

A maior proporção de uso da AE encontrada entre as brasileiras do presente estudo pode ser justificada pela elevada proporção de uso de métodos contraceptivos que dependem primordialmente da disciplina do(a) usuário(a $)^{7}$, como a pí- lula oral e o preservativo, que, por sua vez, estão associados a maiores taxas de descontinuidades contraceptivas, acarretando uso mais frequente da $\mathrm{AE}^{18}$. Pode ser que a possibilidade de comprar o método em farmácias comerciais, mesmo sem 


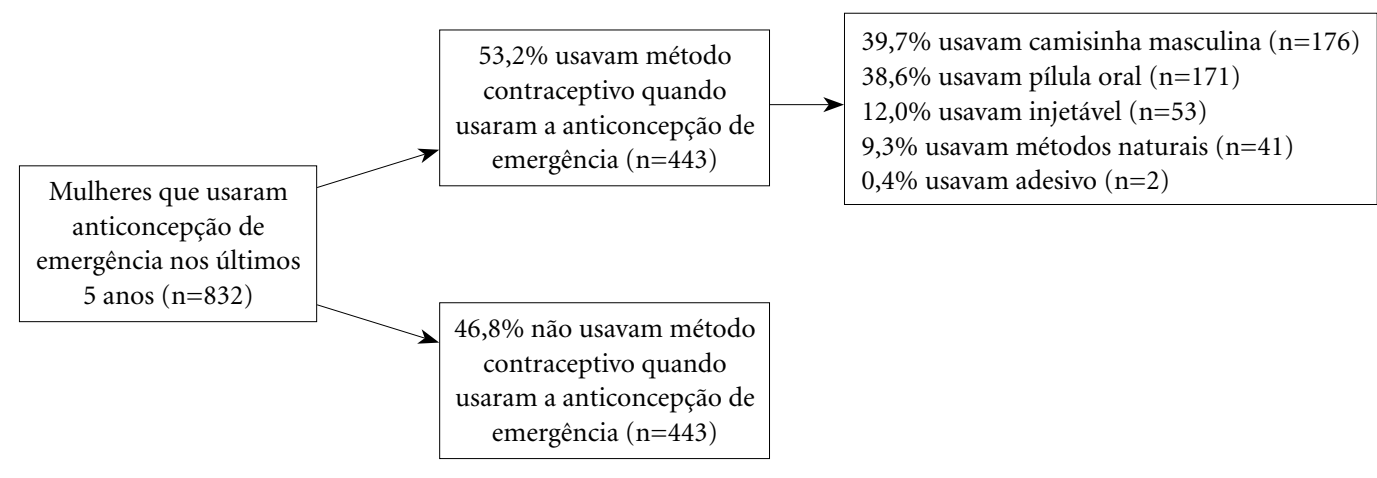

Figura 1. Método contraceptivo em uso quando usou a anticoncepção de emergência. São Paulo-SP, Aracaju-SE e Cuiabá-MT, 2015-2017.

Fonte: Elaborado pelas autoras.
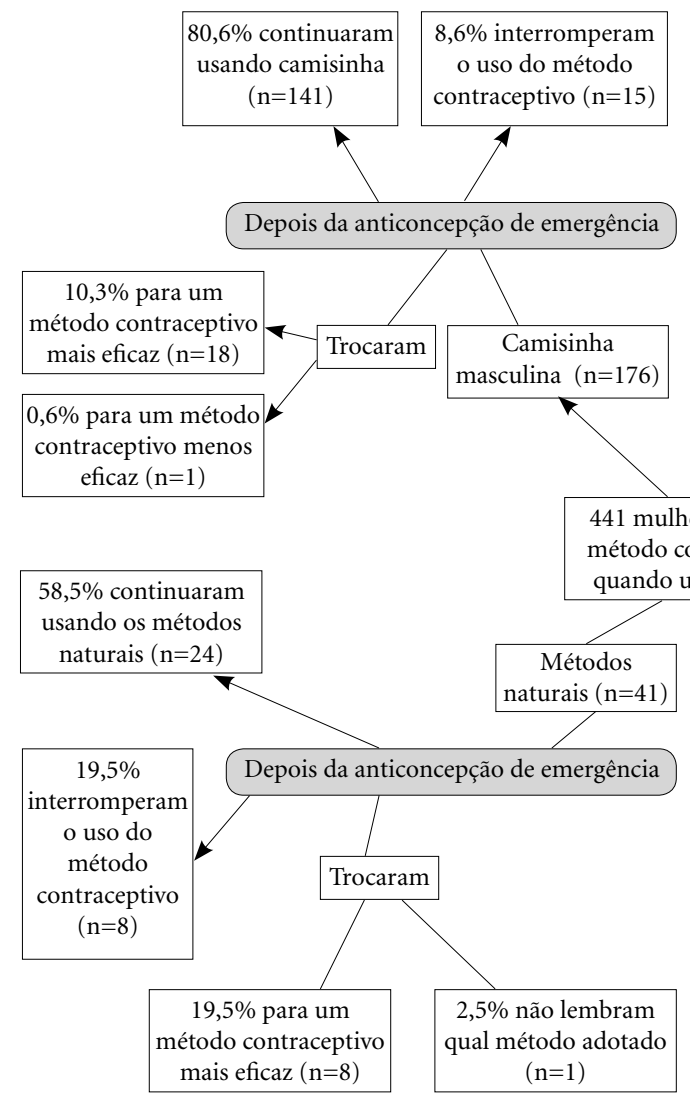

$90,6 \%$ continuaram usando a pílula $(\mathrm{n}=155)$

$4,1 \%$ interromperam o uso do método contraceptivo $(n=7)$
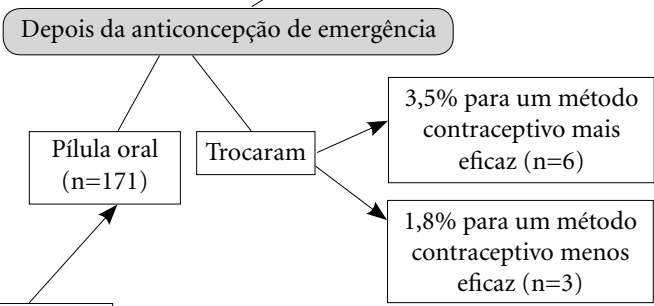

41 mulheres usavam método contraceptivo quando usaram a $\mathrm{AE}$

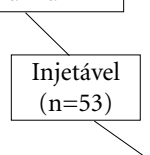

$88,7 \%$ continuaram usando injetável $(\mathrm{n}=47)$

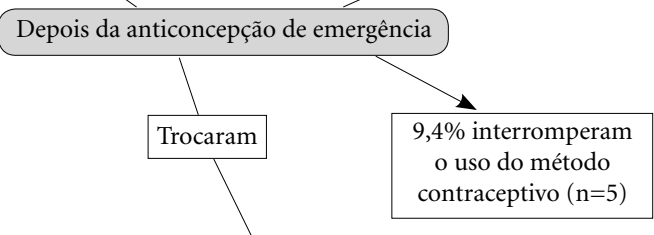

1,9\% para um método

contraceptivo menos eficaz $(n=1)$

Figura 2. Uso de método contraceptivos após o uso da AE. São Paulo-SP, Aracaju-SE e Cuiabá-MT, 2015-2017.

Fonte: Elaborado pelas autoras. 


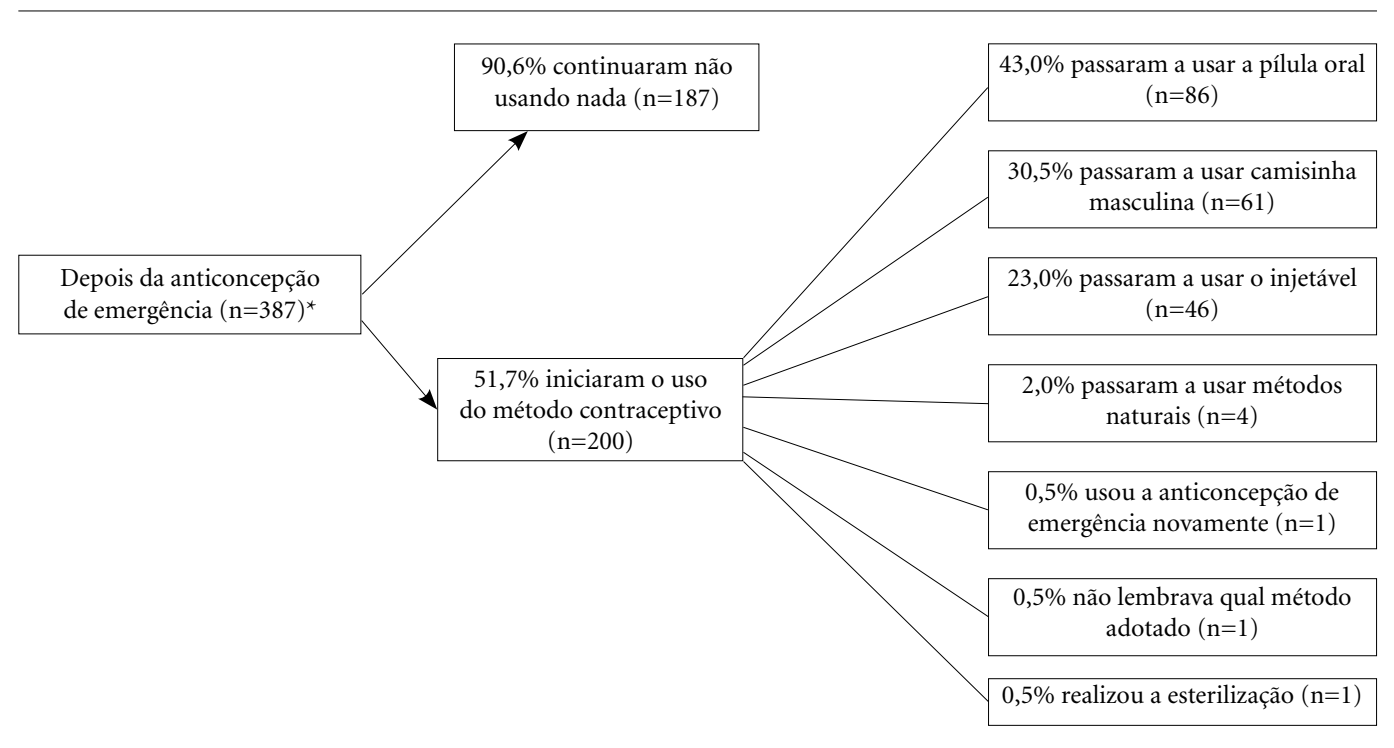

Figura 3. Uso de método contraceptivo após o uso da anticoncepção de emergência entre mulheres que não usavam método. São Paulo-SP, Aracaju-SE e Cuiabá-MT, 2015-2017.

*(n=387): duas mulheres não responderam qual método contraceptivo usaram depois de usar a anticoncepção de emergência.

Fonte: Elaborado pelas autoras.

prescrição médica, também contribua para esse quadro $^{1,10}$.

$\mathrm{Na}$ análise de regressão logística múltipla, os aspectos associados ao uso da AE foram: menor idade, maior escolaridade, maior nível socioeconômico, ter trabalho remunerado, ter tido maior número de parceiros sexuais e estar em união conjugal. A idade associou-se estatisticamente ao uso da AE nas três capitais. Esse achado é consistente com outros estudos ${ }^{17,19}$, ao mostrar que o uso da AE ocorre mais frequentemente entre jovens. Pode ser que as mais jovens troquem mais de métodos contraceptivos a cada transição nos relacionamentos afetivo-sexuais, tanto de um relacionamento para outro como dentro do mesmo relacionamento, ao caminhar para maior estabilidade, e por isso, se deparam com maior necessidade de recorrer à $\mathrm{AE}$ nos momentos de falhas ou inconsistências ${ }^{16}$. Além disso, outros pesquisadores já mostraram que são mais propensas a usar a AE, mulheres com escolaridade mais elevada e pertencentes a extratos socioeconômicos mais altos $^{16,20,21}$, possivelmente por terem maior autonomia e maior possibilidade de acesso ao método, tendo em vista que o principal local de obtenção da AE no Brasil é nas farmácias comerciais ${ }^{10}$. Em relação ao número de parceiros sexuais, outros pesquisadores também constataram que mulhe- res com dois ou mais parceiros sexuais eram as que mais usavam a $\mathrm{AE}$, possivelmente porque a mudança de parcerias influencia a prática contraceptiva, provocando maior troca de métodos, situação que favorece o uso da $\mathrm{AE}^{17,22}$.

Em nosso estudo, as mulheres que estavam em união conjugal mostraram-se menos propensas a usar a AE, quiçá porque nesses contextos a ocorrência de uma gravidez é menos preocupante, mesmo que inesperada, de forma que as mulheres não se preocupam tanto em se prevenir no caso de uma relação sexual desprotegida ${ }^{13}$. A flexibilidade no uso de contraceptivos quando o parceiro é conhecido e o relacionamento se torna estável já é bem descrita ${ }^{23}$. Além disso, é provável que as mulheres que não vivem com o parceiro tenham mais relações sexuais esporádicas, o que as colocaria em situações de maior vulnerabilidade para o sexo desprotegido e, consequentemente à maior necessidade de usar a AE. Segundo Raine et al. $^{24}$, há pouca confiança, comunicação e intimidade entre os parceiros nas relações eventuais, o que dificulta a tomada de decisões contraceptivas, acarretando em maior uso da AE neste tipo de relacionamento.

A análise da dinâmica contraceptiva antes e após o uso da AE mostrou que pouco mais da metade das mulheres usava método contracepti- 
vo quando usou a AE. O principal método utilizado era a camisinha masculina e a pílula oral, métodos que, segundo Polis et al..$^{25}$, apresentam altas taxas de descontinuidade, provavelmente devido ao fato da camisinha masculina ser um método de curta duração, usado geralmente em relacionamentos casuais ou no início dos relacionamentos ${ }^{26}$; ao passo que a inconsistência no uso da pílula oral está mais relacionada aos esquecimentos ou por conta dos seus efeitos colaterais ${ }^{27}$.

Após o uso da AE, a maioria das mulheres continuou a utilizar o mesmo método contraceptivo. As mulheres que usavam pílula oral e injetável foram as que mais continuaram a utilizar o mesmo método após o uso da AE. Embora não tenham descrito o tipo de método contraceptivo utilizado, Alano et al. ${ }^{11}$ encontraram resultado semelhante, em que 97,8\% das entrevistadas declararam que não houve troca/abandono do método regular após o uso da AE. É curioso o fato de que, dentre as mulheres que usavam métodos naturais e que usaram a AE, poucas passaram a usar um método contraceptivo mais eficaz posteriormente, embora tenham reconhecido que tiveram uma relação sexual desprotegida, tenham se lembrado da AE e conseguido usá-la. Isso pode se dever ao fato que tais mulheres usavam os métodos naturais porque não conseguiram acessar outros métodos contraceptivos, nem mesmo após o uso da $\mathrm{AE}$, ou porque incorporaram os métodos naturais em busca de bem-estar e maior controle sobre o próprio corpo ${ }^{28}$. Dessa forma, o uso de métodos naturais pode vir a ter um incremento em grupos de mulheres jovens e escolarizadas que não se adaptam a métodos frequentemente ofertados nos serviços de saúde, não se devendo apenas a uma mera falta de opção, o que se constitui em lacuna de conhecimento na área de planejamento reprodutivo.

Nossos achados mostraram, pois, que o uso da AE não contribuiu para que a maioria das mulheres interrompesse ou trocasse o método contraceptivo em uso, o que reitera resultados de outros estudos, como o de Rowlands et al. ${ }^{29}$, Jackson et al. ${ }^{30}$ e Raine et al. ${ }^{31}$, que já haviam mostrado que as mulheres com fornecimento prévio (advanced provision) ou acesso ampliado à $\mathrm{AE}$ não possuem maior probabilidade de abandonar o método em uso e nem de trocá-lo para métodos menos eficazes $^{29-31}$. Cenário semelhante foi encontrado entre universitárias brasileiras, em que a maioria delas não mudou seu método após o uso da $\mathrm{AE}^{32}$.

Praticamente metade das mulheres não usava método contraceptivo quando usou a $\mathrm{AE}$, situação que chama a atenção, porque indica reco- nhecimento de terem vivenciado uma relação sexual desprotegida e de estarem vulneráveis a uma gravidez, que as levou a utilizar a AE. Nesse contexto, é interessante notar que, dessas, mais da metade passou a usar método contraceptivo, principalmente a pílula oral, a camisinha masculina e o injetável, o que reafirma que para muitas mulheres, a AE atua como um precursor do uso de contracepção regular, fenômeno que vem sendo denominado como "bridging", isto é, a AE pode ser uma "ponte" para o uso da contracepção regular ${ }^{33}$. Igualmente, outros estudos verificaram que mulheres que não usavam nenhum método contraceptivo adotaram um método de uso regular após o uso da $\mathrm{AE}^{26,34,35}$. Dessa forma, embora a AE não seja uma opção segura para substituir método contraceptivo de uso regular, ela pode propiciar a transição para o uso de contracepção mais eficaz e consistente ${ }^{36}$.

Notou-se que nenhuma mulher iniciou o uso do DIU ou outro método contraceptivo de longa duração após o uso da AE, talvez pelo fato de ainda haver barreiras para a disponibilização do DIU no SUS ${ }^{37}$ e que implantes hormonais sequer estão disponíveis nas UBS.

Por sua vez, os dados também apontam para a necessidade de atenção mais qualificada para as mulheres que não usam método contraceptivo, mas que são capazes de reconhecer uma relação sexual desprotegida, tanto que a AE é indicada justamente nesses casos, pois é no momento em que essas mulheres buscam a AE que o profissional de saúde tem a oportunidade de orientá-las, como também de ofertar outros métodos que contribuam para minimizar os riscos de ocorrência de gravidez não intencional, utilizando a AE como "porta de entrada" para tal prevenção.

Uma das potencialidades deste estudo foi compreender a dinâmica contraceptiva após o uso da AE entre um grupo de mulheres vulneráveis à gravidez não intencional em três capitais brasileiras. A compreensão dessa dinâmica pode auxiliar no aprimoramento de programas para mulheres nos serviços da Atenção Primária à Saúde no Brasil, principalmente no que se refere ao aumento da disponibilidade do método, uma vez que a $\mathrm{AE}$ não interfere no uso rotineiro de métodos contraceptivos.

No entanto, o estudo apresenta algumas limitações, como o fato de que a amostra representa apenas parcela de mulheres atendidas pelo SUS; o fato de não ter sido analisado o tempo exato decorrido entre o uso da AE e o início do método contraceptivo adotado posteriormente. Também não se perguntou o motivo pelo qual as mulhe- 
res usaram a $\mathrm{AE}$, porém tais motivos, que convergem com a sua indicação, já são amplamente conhecidos: relação sexual desprotegida, falha ou uso inadequado de um método contraceptivo ${ }^{12,38}$. Ademais, por se tratar de um estudo com informações autorreferidas sobre a história sexual e o uso de métodos contraceptivos que ocorreram no passado, há que se considerar possíveis vieses de memória. Contudo, a análise da dinâmica contraceptiva antes e após o uso da $\mathrm{AE}$ foi realizada somente com as mulheres que relataram seu uso nos cinco anos anteriores à entrevista, com vistas a minimizar o viés de memória. Por fim, há que se considerar a não exploração do tipo de parceria no momento do uso da $\mathrm{AE}$, questão importante, uma vez que a parceria pode influenciar o uso de métodos contraceptivos pelas mulheres ${ }^{39}$.

\section{Conclusão}

O perfil das mulheres que usou a AE mostrou-se semelhante nas diferentes capitais brasileiras estudadas, São Paulo-SP, Aracaju-SE e Cuiabá-MT. Constatou-se que o método é majoritariamente utilizado por mulheres jovens, mais escolarizadas, que trabalham, que não estão em união está- vel e que tiveram dois ou mais parceiros sexuais na vida. De forma geral, a AE tem sido utilizada pelas mulheres brasileiras e amplamente por aquelas que frequentam os serviços públicos de saúde, garantindo o exercício de seus direitos sexuais e reprodutivos, ao permitir a prevenção de gestações não intencionais.

$\mathrm{O}$ uso da $\mathrm{AE}$ não provocou troca ou interrupção do método contraceptivo em uso para a maioria absoluta das mulheres. Na verdade, a $\mathrm{AE}$ representou um grande potencial para atuar como ponte para o início de uma proteção contraceptiva efetiva entre as mulheres que não utilizavam método contraceptivo, razão pela qual este método deveria ser melhor difundido e sua oferta ampliada nos serviços de saúde. Ainda é preocupante que metade das mulheres que não usava qualquer método continuasse sem adotar nenhum, mesmo após usar a AE. Esse grupo vulnerável à gestação não intencional merece mais atenção por parte dos serviços de atenção básica à saúde, seja por meio de um melhor diálogo durante a oferta da própria $\mathrm{AE}$, seja por meio de melhor divulgação dos outros métodos regulares, dando ênfase também aos métodos de longa duração, como o DIU de cobre, disponibilizado nas UBS do Sistema Único de Saúde. 


\section{Colaboradores}

ALV Borges coordenou todo o projeto de pesquisa, desde a concepção, financiamento, coleta de dados, análise dos dados até a elaboração e revisão do artigo. RFS Gonçalves conduziu a análise dos dados e atuou na elaboração e revisão do artigo. CBN Chofakian participou da elaboração e revisão do artigo. NC Nascimento participou da elaboração e revisão do artigo. RMMD Figueiredo participou da elaboração e revisão do artigo. OA Santos atuou na concepção do estudo e na supervisão da coleta de dados; além de ter participado da revisão do artigo. E Fujimori participou da elaboração e revisão do artigo. EA Divino atuou na supervisão da coleta de dados e revisão do artigo.

\section{Referências}

1. Pacheco A, Costa AR, Martins I, Palma F, Sousa F, Almeida MC, Bombas T. Recomendações sobre Contraceção de Emergência. Coimbra: Sociedade Portuguesa da Contracepção; 2015.

2. Westley E, Bass J, Puig C. A global assessment of emergency contraception acessibility. In: Figueiredo R, Borges ALV, Paula SHB, organizadoras. Panorama da Contracepção Emergência no Brasil. São Paulo: Instituto de Saúde; 2016. p.24-38.

3. Brasil. Ministério da Saúde (MS). Anticoncepção de emergência: perguntas e respostas para profissionais de saúde. Brasília: MS; 2011.

4. International Consortium for Emergency Contraception (ICEC) [Internet]. [acessado 2020 jan 20]. New York: ICEC; 2017. Disponível em: https://www.cecinfo.org/.

5. Krassovics M, Viragh G. Usage patterns and attitudes towards emergency contraception: the International Emergency Contraception Research Initiative. Eur J Contracept Reprod Health Care 2016; 21(4):310-317.

6. Brasil. Ministério da Saúde (MS). Protocolo para Utilização do Levonorgestrel. Brasília: MS; 2013.

7. Brasil. Ministério da Saúde (MS). Secretaria da Ciência, Tecnologia e Insumos Estratégicos. Departamento de Ciência e Tecnologia. PNDS: 2006: Pesquisa Nacional de Demografia e Saúde da Criança e da Mulher: Relatório. Brasília: MS; 2008.

8. Figueiredo R, Pupo LR, Alves MCGP, Escuder MML Comportamento sexual, uso de preservativos e contracepção de emergência entre adolescentes do município de São Paulo. Boletim Instituto de Saúde 2008; 46:33-31.

9. Chofakian CBN, Borges ALV, Fujimori E, Hoga LAK. Conhecimento sobre anticoncepção de emergência entre adolescentes do Ensino Médio de escolas públicas e privadas. Cad Saude Publica 2014; 30(7):15251536.

10. Olsen JM, Lago TG, Kalckmann S, Alves MCGP, Escuder MML. Práticas contraceptivas de mulheres jovens inquérito domiciliar no Município de São Paulo, Brasil. Cad Saude Publica 2018; 34(2):e00019617.

11. Alano GM, Costa LN, Miranda LR, Galato D. Conhecimento, consumo e acesso à contracepção de emergência entre mulheres universitárias no sul do Estado de Santa Catarina. Cien Saude Colet 2012; 17(9):23972404.

12. Borges ALV, Fujimori E, Hoga LAK, Contin MV. Práticas contraceptivas entre jovens universitárias: o uso da anticoncepção de emergência. Cad Saude Publica 2010; 26(4):816-826.

13. Santos OA, Borges ALV, Chofakian CBN, Pirotta KCM. Determinants of emergency contraception non-use among women in unplanned or ambivalent pregnancies. Rev Esc Enferm 2014; 48(esp.):16-22.

14. Carmo MSAG, Duarte SFP. Perfil das usuárias de anticoncepcionais de emergência: uma revisão sistemática. Id Line Multidiscip Psycology J 2017; 11(35):324317.

15. Han L, Saavedra-Avendano B, Lambert W, Fu R, Rodriguez MI, Edelman A, Darney B. Emergency contraception in Mexico: trends in knowledge and ever-use 2006-2014. Matern Child Health J 2017; 21(11):21322139. 
16. Daniels K, Jones J, Abma JC. Use of emergency contraception among women aged 15-44, United States, 2006-2010. NCHS Data Brief 2013; (112):1-8.

17. Moreau C, Bajos N, Trussell J. The impact of pharmacy access to emergency contraceptive pills in France. Contraception 2006; 73(6):602-608.

18. Moreau C, Trussell J, Michelot F, Bajos N. The effect of access to emergency contraceptive pills on women's use of highly effective contraceptives: results from a French National Cohort Study. Am J Public Healh 2009; 99(3):441-442.

19. Goulard H, Moreau C, Gilbert F, Job-Spira N, Bajos N. Contraceptive failures and determinants of emergency contraception use. Contracept 2006; 74(3):208-213.

20. Díaz SHE, Alvarado G, Ezcurra E. Acceptability of emergency contraception in Brazil, Chile, and Mexico. 2 - Facilitating factors versus obstacles. Cad Saude Publica 2003; 19(6):1729-1737.

21. Williamson LM, Buston K, Sweeting H. Young women's perceptions of pregnancy risk and use of emergency contraception: findings from a qualitative study. Contraception 2009; 79(4):310-315.

22. Waltermaurer E, Doleyres HM, Bednarczyk RA, McNutt L-A. Emergency contraception considerations and use among college women. J Womens Health 2013; 22(2):141-146.

23. Pirotta KCM, Schor N. Intenções reprodutivas e práticas de regulação da fecundidade entre universitários. Rev Saude Publica 2004; 38(4):495-502.

24. Raine TR, Gard JC, Boyer CB, Haider S, Brown BA, Ramirez Hernandez FA, Harper CC. Contraceptive decision-making in sexual relationships: young men's experiences, attitudes and values. Cult Health Sex 2010; 12(4):373-386.

25. Polis CB, Bradley SEK, Bankole A, Onda T, Croft T, Singh S. Typical-use contraceptive failure rates in 43 countries with Demographic and Health Survey data: summary of a detailed report. Contraception 2016; 94(1):11-17.

26. Manlove J, Welti K, Wildsmith E, Barry M. Relationship types and contraceptive use within young adult dating relationships. Perspect Sex Reprod Health 2014; 46(1):41-50.

27. Moreau C, Trussell J, Bajos N. The determinants and circumstances of use of emergency contraceptive pills in France in the context of direct pharmétodo contraceptivoy access. Contraception 2006; 74(6):476-482.

28. Magalhães AC, Pereira DSA, Jardim DBB, Caillaux M, Sales VBL. Vivência da mulher na escolha do Método de Ovulação Billings. Rev Bras Enferm 2013; 66(4):485-492.

29. Rowlands S, Devalia H, Lawrenson R, Logie J, Ineichen $\mathrm{B}$. Repeated use of hormonal emergency contraception by younger women in the UK. BMJ Sex Reprod Health 2000; 26(3):138-143.

30. Jackson RA, Schwarz EB, Freedman L, Darney P. Advance supply of emergency contraception: effect on use and usual contraception - a randomized trial. Obstet Gynecol 2003; 102(1):8-16.
31. Raine TR, Harper CC, Rocca CH, Fischer R, Padian N, Klausner JD, Darney PD. Direct access to emergency contraception through pharmacies and effect on unintended pregnancy and STIs: a randomized controlled trial. J Am Med Assoc 2005; 293(1):54-62.

32. Chofakian CBN, Moreau C, Borges ALV, Santos OA. Contraceptive patterns after use of emergency contraception among female undergraduate students in Brazil. Eur J Contracep Rep Health Care 2018; 23(5):335-343.

33. Cameron S, Glasier A. The need to take a 'new look' at emergency contraception. J Fam Plann Reprod Health Care 2010; 36(1):3-4.

34. Mosher WD, Jones J. Use of Contraception in the United States: 1982-2008. National Center for Health Statistics. Vital Health State 2010; 29:1-44.

35. Cameron ST, Glasier A, Johnstone A, Rae L. Ongoing contraception after use of emergency contraception from a specialist contraceptive service. Contraception 2011; (4):368-371.

36. Trussell J, Raymond E, Cleland K. Emergency contraception: A last chance to prevent unintended pregnancy. Contemporary Readings Law Social Justice 2014; 6(2):7-38.

37. Gonzaga VAS, Borges ALV, Santos OA, Rosa PLFS, Gonçalves RFS. Barreiras organizacionais para disponibilização e inserção do dispositivo intrauterino nos serviços de atenção básica à saúde. Rev Esc Enferm USP 2017; 51:e03270.

38. Moreau C, Bouyer J, Gilbert F, Group TC, Bajos N. Social, demographic and situational characteristics associated with inconsistent use of oral contraceptives: evidence from France. Perspect Sex Reprod Health 2006; 38(4):190-196.

39. Donadiki EM, Jimenez-Garcıa R, Velonakis EG, Hernandez-Barrera V, Sourtzi P, Lopez de Andres A, Jimenez-Trujillo I, Gallardo Pino C, Carrasco-Garrido P. Factors related to contraceptive methods among female higher education students in Greece. J Pediatr Adolesc Gynecol 2013; 26(6):334-339.

Artigo apresentado em 29/05/2019

Aprovado em 09/12/2019

Versão final apresentada em 11/12/2019

Editores-chefes: Romeu Gomes, Antônio Augusto Moura da Silva 